\title{
Strain-accelerated dynamics of soft colloidal glasses
}

\author{
Praveen Agarwal and Lynden A. Archer* \\ Department of Chemical and Biomolecular Engineering, Cornell University, Ithaca, New York 14850, USA \\ (Received 12 October 2010; revised manuscript received 14 December 2010; published 11 April 2011)
}

\begin{abstract}
We have investigated strain-accelerated dynamics of soft glasses theoretically and experimentally. Mechanical rheology measurements performed on a variety of systems reveal evidence for the speeding-up of relaxation at modest shear strains in both step and oscillatory shear flows. Using the soft glassy rheology (SGR) model framework, we show that the observed behavior is a fundamental, but heretofore unexplored attribute of soft glasses.
\end{abstract}

DOI: 10.1103/PhysRevE.83.041402

PACS number(s): 82.70.Dd, 83.80.Ab, 83.80.Hj

\section{INTRODUCTION}

The concept of deformation-accelerated dynamics in soft matter dates back to the seminal work of Eyring in the 1930s on polymeric glasses [1]. The central idea is that deformation lowers the energy barrier for molecular motions, enhancing molecular mobility of the glass phase. Within the last decade, there have been significant advances in the characterization tools for studying the effect of deformation on dynamics, which has lead to accumulating evidence of stress, strain, and strain-rate accelerated relaxation in polymeric glasses. Lee et al. [2], for example, used a photobleaching technique to probe the molecular mobility of poly(methyl methacrylate) (PMMA) during uniaxial elongation and reported more than a 1000-fold increase in molecular mobility relative to the unstretched polymer. These authors reported that more than a 1000-fold increase in molecular mobility of PMMA is produced by extension. More recently, Warren et al. [3] studied relaxation dynamics in deformed polymer glasses using molecular dynamic simulations. They found that the macroscopic strain is the key variable controlling the acceleration of relaxation dynamics, independent of deformation protocol or aging time.

There has been considerable interest in studying colloids as model systems to understand the glass transition [4,5]. Colloidal glasses are now generally understood to exist in metastable structural states in which individual material elements (particles) are trapped in potential energy wells deeper than thermal energy $[6,7]$. In these systems, deformation is required to achieve any structural relaxation [6-8]. In laponite suspensions, Joshi et al. [9] reported that an external shear stress produces an effect on structural relaxation analogous to temperature. Recently we reported that in large-amplitude oscillatory shear, the shear strain could be used, in an analogous fashion to temperature in polymers, to dramatically extend the dynamic range accessible in experiments [10]. Termed time-strain superposition (TSS), this process is similar to the strain-rate frequency superposition (SRFS) methodology reported by Wyss et al. [11], wherein the strain rate is the variable used to extend the experimental time window. In this letter we report direct evidence of strain-accelerated relaxation dynamics in soft glasses. We further show that these observations are consistent with predictions based on the soft glassy rheology (SGR) model, and are, in fact, central to the recent discovery of TSS in soft glasses [10].

\footnotetext{
*gmlaa25@gmail.com
}

\section{MATERIALS AND METHODS}

This report focuses on "self-suspended" suspensions [10, 12] created by tethering short polyisoprene (PI) chains to silica nanoparticles. Termed nanoscale ionic materials (NIMs), these materials are attractive because they display fluid-like properties even in the absence of an external solvent. This feature arises in a straightforward way from the abundance of polymeric fluid tethered to the nanoparticles, but makes NIMs exceptionally stable against aggregation and, consequently, good models for soft glasses [10]. Additionally, because the particles and suspending medium (tethered polymer) are permanently coupled, NIMs have recently been shown by theory to be characterized by a limiting equilibrium structure factor $S(q \rightarrow 0)=0$ [12] (i.e., consistent with the notion that each nanoparticle carries around a share of its suspending fluid tethered to its surface).

To characterize relaxation dynamics of NIMs, mechanical rheology measurements were performed at a temperature of $25^{\circ} \mathrm{C}$ in step and oscillatory shear using Rheometrics Scientific ARES and Anton Paar MCR 501 rheometers equipped with cone-and-plate fixtures. Before every measurement, the sample was presheared by applying a large amplitude oscillatory shear strain sweep ranging from $0.01 \%$ to $200 \%$ at a frequency of $10 \mathrm{rad} / \mathrm{s}$ to erase any history due to loading or previous deformations. After the preshear, a waiting time maintained large, in comparison to the measurement time, was allowed before performing rheology measurements.

Results reported in this report are for a single NIMs material comprised of $10 \pm 2 \mathrm{~nm}$ diameter silica $\left(\mathrm{SiO}_{2}\right)$ nanoparticle cores and a PI corona of molecular weight $5000 \mathrm{~g} / \mathrm{mol}$. The volume fraction $\phi$ of silica in these $\mathrm{SiO}_{2}$-PI NIMs is 0.12 . To ensure that the experimental results are not specific to a particular material, we have carried out similar experiments on two other materials: (i) $\mathrm{NIMs}$ with $\mathrm{SiO}_{2}$ core and polyethylene glycol (PEG) corona; and (ii) suspensions created by dispersing PEG tethered hairy nanoparticles in PEG oligomer hosts. Results for these two systems are provided as supplementary information.

\section{EXPERIMENTAL RESULTS}

A wide variety of materials including foams, emulsions, suspensions, and star polymers with crowded branch points have been reported to display universal rheological features such as slow dynamics, yielding, and aging, understood to originate from metastability and disorder. These materials 

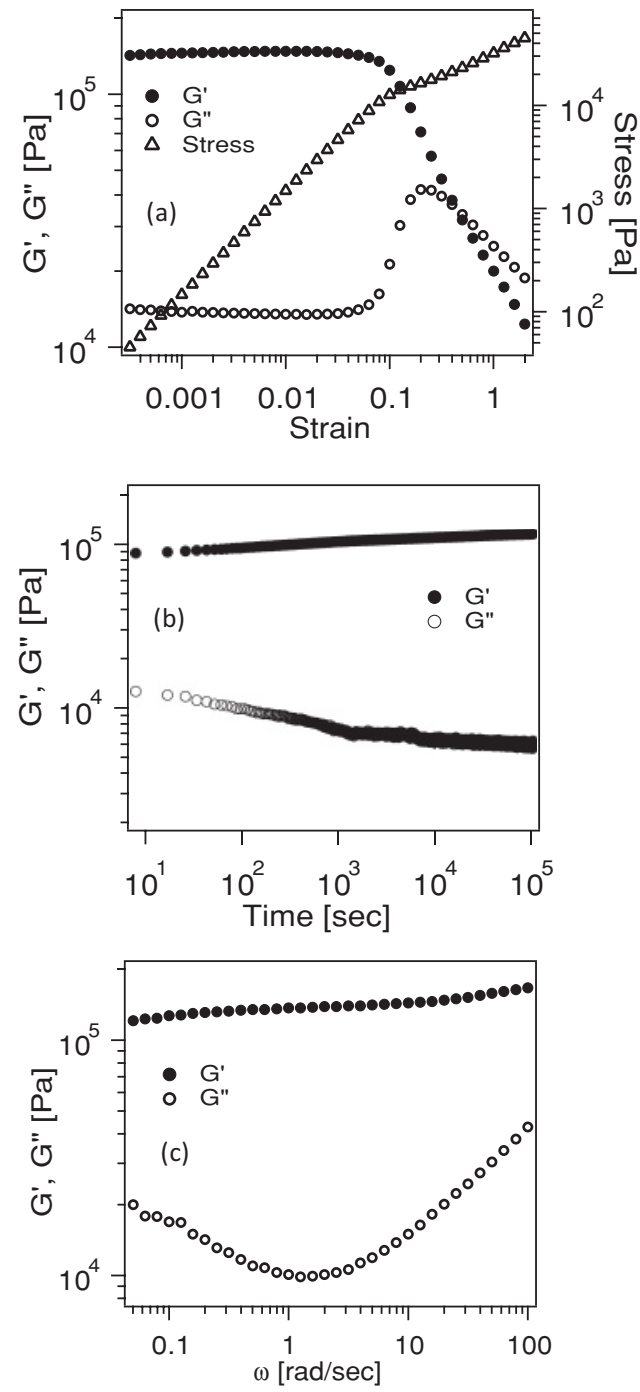

FIG. 1. (a) Stain sweep measurements at a frequency of 10 $\mathrm{rad} / \mathrm{sec}$. (b) $\mathrm{G}^{\prime}$ and $\mathrm{G}^{\prime \prime}$ as a function of time after preshear by strain sweep ranging from $0.01 \%$ to $200 \%$. After the preshear, small-amplitude oscillatory shear $(\gamma=0.5 \%$ and $\omega=5 \mathrm{rad} / \mathrm{s})$, was used to record moduli as a function of time. (c) Frequency sweep at $\gamma=0.5 \%$.

have been collectively termed "soft glasses" [5-7]. Figure 1(a) shows the effect of shear strain on the storage $\left(G^{\prime}\right)$ and loss $\left(G^{\prime \prime}\right)$ modulus of the $\mathrm{SiO}_{2}$-PI NIMs. All of the features displayed, including the $G^{\prime}$-dominant linear viscoelastic regime followed by a pronounced loss maximum prior to the onset of strain softening, are consistent with expectations for soft glasses [5-7,10,11,13,14]; for which this behavior is attributed to strain-induced breakage of collections of structural units termed "cages," which constrain motion of individual particles in the material.

One complication that arises in the study of soft glassy materials is that their properties change with time. Termed "aging," this phenomenon is understood to reflect the slow densification of the material as its elements sample progressively deeper potential wells in their energy landscape [7]. Figure 1(b) shows the time evolution of the viscoelastic moduli of the $\mathrm{SiO}_{2}$-PI NIMs just after preshear. It can be clearly seen from the plot that there is no significant change in the values of both $G^{\prime}$ and $G^{\prime \prime}$ with time, signifying that the effect of aging in these systems is small. Figure 1(c) shows the linear viscoelastic response of the same material as a function of shear frequency. The response is again seen to be typical for a soft glass $[5-7,11,13,14]$. The upturn in the loss modulus observed at low frequency is common for many soft glasses [5-7,11,13]; it appears to presage a structural relaxation process outside of the frequency window accessed by the experiment.

As reported previously for nanoparticle suspensions [15], measurement of the relaxation modulus $G(t)=\sigma(t) / \gamma$ following the application of a step strain $\gamma(t)=\gamma H(t)$ where $\sigma$ is stress and $H(t)$ is a step function, provides a straightforward method for extending the dynamic range of the oscillatory shear experiment and provides the most direct comparisons of experimental data with SGR model predictions [16]. Prior to each step-strain measurement, the material was presheared using large-amplitude oscillatory shear and rested for a period of 5000 seconds. Figure 2(a) shows $G(t)$ at multiple shear strains for the first 500 seconds following imposition of the step strain. The observation time is limited to ensure that the measurement time is much smaller than the resting or waiting time $[17,18]$.
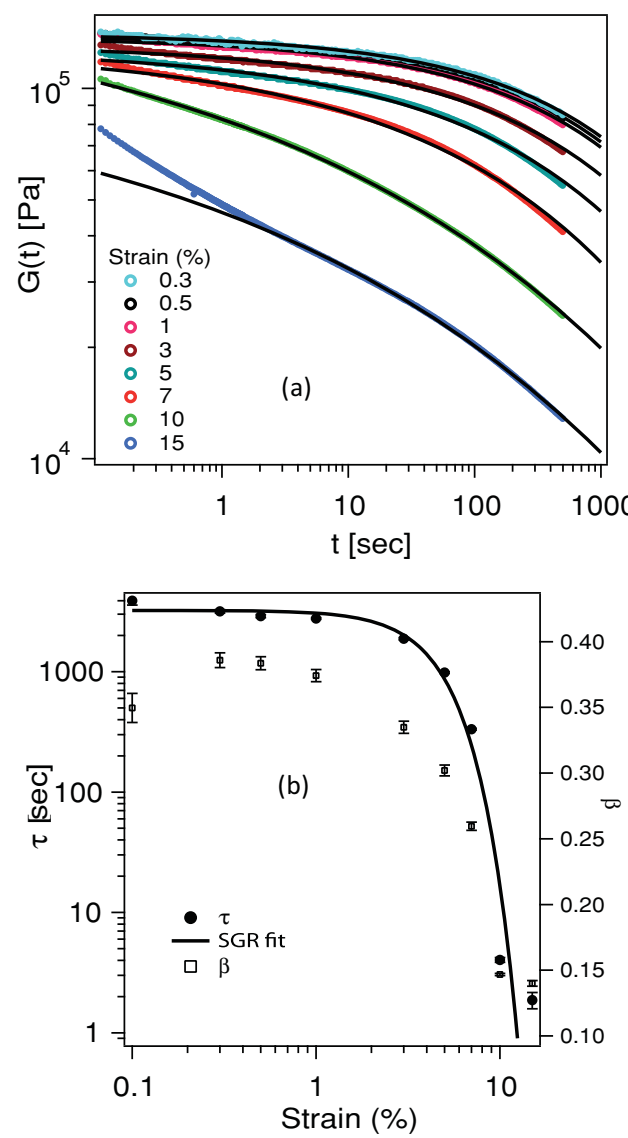

FIG. 2. (Color) (a) Relaxation modulus vs. time for increasing strain (from top to bottom), decay of the modulus is faster at higher strains, lines are fit to KWW expression. (b) Parameters $\tau$ and $\beta$ obtained from KWW fits for different strains; the characteristic time $\tau$ decreases with strain and $\beta$ shows slight decrease with strain. Solid line is the fit using Eq. (2) with $\tau_{0}=3230 \pm 156 \mathrm{~s}$ and $k=526 \pm$ 105. 
It is readily apparent from Fig. 2(a) that the long-time decay of $G(t)$ speeds up considerably with increasing shear strain. The continuous lines in the figure indicate that the stress relaxation is of the stretched exponential or KohlrauschWilliam-Watts (KWW) form [2,19-22]

$$
G(t)=A \exp \left(-\left(\frac{t}{\tau}\right)^{\beta}\right) .
$$

Where $A$ is the zero time or baseline value of the relaxation modulus, $t$ is the elapsed time after the step, $\tau$ is a characteristic material relaxation time, and $\beta$ is defined as the coupling parameter. The characteristic time $\tau$ and coupling parameter $\beta$ deduced from the fits are provided in Fig. 2(b). It can be seen from the figure that $\tau$ is constant at low strains, indicative of the linear viscoelastic regime and that it decreases substantially at high strains, consistent with visual observation, as well as with the earlier reports by Lee et al. [2] and Warren et al. [3] for polymeric glasses. The exponent $\beta$ decreases with shear strain, but the change is more modest than reports for polymeric glasses [2,3], where the distribution of relaxation times is narrowed markedly by increasing the shear strain. The solid line in Fig. 2(b) is fit using the equation

$$
\tau=\tau_{0} \exp \left(-k \gamma^{2}\right)
$$

with $\tau_{0}=3230 \pm 156 \mathrm{~s}$ and $k=526 \pm 105$, where $\tau_{0}$ is the relaxation time under very small strain and $k$ is a constant.

Recently Bandopadhyay et al. [19] reported that $G(t)$ deduced from step-strain rheological measurements in laponite suspensions relaxes via a two-step (fast, followed by slow) process. It is apparent from Fig. 2(a) that a similar two-step relaxation is seen in our $\mathrm{SiO}_{2}$-PI NIMs and that the fast relaxation mode first appears at a strain of $15 \%$, that is, above the yield strain [the yield strain is determined from Fig. 1(a) from stress versus strain data as the strain at which stress versus strain becomes nonlinear]. At shear strains beyond the yield strain it is not possible to fit the entire $G(t)$ versus $t$ plot using the KWW function. We have therefore probed dynamics at these higher strains using oscillatory shear rheology measurements.

If strain accelerates structural dynamics of soft glasses, as is evident from our step shear measurements, the frequency at which the storage and loss modulus intersect or crossover [23] should become progressively more accessible in a frequency sweep experiment performed at higher shear stain. Figure 3 shows that this is indeed the case for our materials, and that the crossover from a $G^{\prime}$ dominant to a $G^{\prime \prime}$-dominant dynamic mechanical response occurs at higher oscillation frequency as the strain is increased. The figure also shows that the value of the modulus at the crossover frequency remains essentially unaffected by strain; an indication that the principal effect of strain is to accelerate relaxation dynamics.

\section{THEORETICAL RESULTS}

The soft glassy rheology model (SGR) proposed by Sollich et al. [6,7] provides a theoretical framework for understanding the phenomena revealed by our experiments. For a step-shear strain of amplitude $\gamma$, this model predicts

$$
\sigma(t)=\gamma G_{\mathrm{eq}}\left(e^{\frac{\gamma^{2}}{2 x}} t\right) .
$$

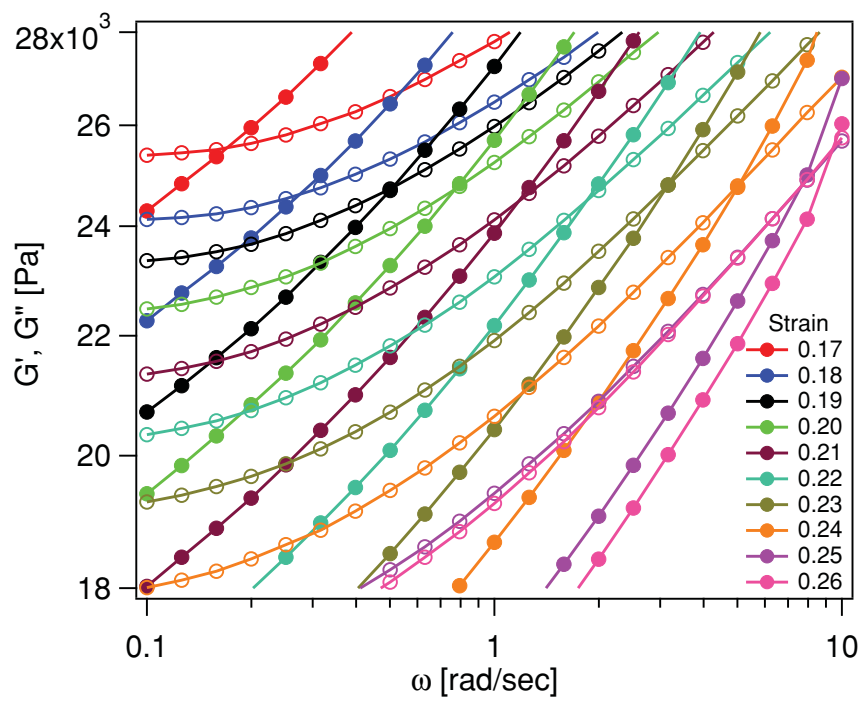

FIG. 3. (Color) Storage and loss modulus vs. frequency at different shear strains. Contrary to observations for the linear viscoelastic regime, the crossover frequency, at which $G^{\prime}=G^{\prime \prime}$, increases with increasing shear strain.

Thus, the relaxation modulus $G(t)$ is given by

$$
G(t)=\frac{\int d E \rho(E) \exp \left(\frac{E}{x}\right) \exp \left(-\exp \left(\frac{\gamma^{2}}{2 x}\right) t \exp \left(-\frac{E}{x}\right)\right)}{\int d E \rho(E) \exp \left(\frac{E}{x}\right) .}
$$

Here $\rho(\mathrm{E})$ is the probability density of yield energies; $x$ is the so-called noise temperature, which describes the typical energy available for hopping; $E$ is the yield energy; and $G_{\text {eq }}$ is a function describing the decay of the relaxation modulus with time $t$.

Substituting $\rho(E)=\exp (-E)$ and integrating Eq. (4) for different shear strains leads to the relaxation moduli reported in Fig. 4(a). As was the case for the experimental results, it is again apparent that the $G(t)$ plots obtained at high strain are shifted toward lower times; implying that at high strain it takes a smaller time for $G(t)$ to decay to the same value as at lower strains. It is also apparent from these plots that the shape of the relaxation modulus at different strains is self similar, implying that $G(t)$ obtained at discrete shear strains can be superimposed to form a master curve as shown in Fig. 4(b). The prediction of time-strain superposition using the SGR model is consistent with our recent experimental discovery of the same phenomenon in soft glassy NIMs [10].

Superposition in Fig. 4(b) is obtained by shifting the curves on the time axis by a shift factor $a_{\gamma}$, defined by the relation

$$
G\left(\gamma, t a_{\gamma}\right)=G(0, t)
$$

The functional form of the strain-dependent shift factor $a_{\gamma}$ can also be deduced from the SGR model by fitting the shift factors required to achieve superposition [inset Fig. 4(b)] to give

$$
a_{\gamma}=\exp \left(-\frac{\gamma^{2}}{2 x}\right)
$$



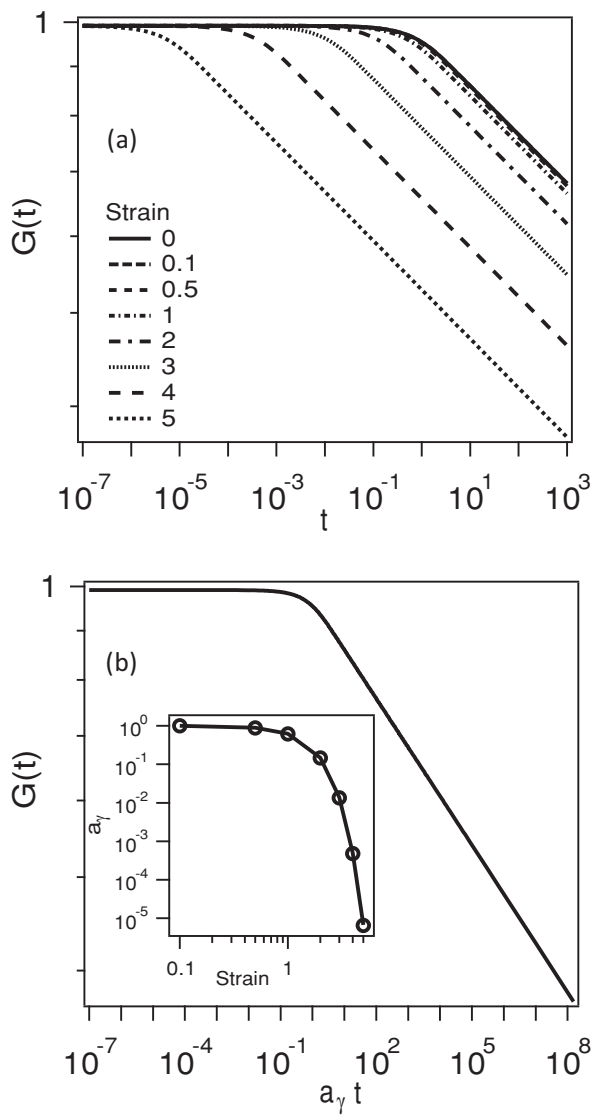

FIG. 4. (a) Relaxation modulus vs. time for different strains calculated using the SGR model. At higher strains, $G(t)$ decays to the same value in a smaller time. (b) Master curve produced by superposition of the curves shown in (a); inset shows shift factors vs. strain, solid line is obtained using the expression given by Eq. (6).

This functional form is, unsurprisingly, the same as the factor multiplying the time scale in Eq. (3). The shift factor $a_{\gamma}$ in Eq. (5) therefore describes the shifting of the time scale in the SGR model due to the application of strain. The form of the shift factor [Eq. (6)] is also strikingly similar to Eq. (2), which describes the effect of strain on the relaxation time obtained from $G(t)$. The analysis reported here uses a value of the noise temperature $x=1.05$ and can be extended to the case $x<1$ following the framework provided by Fielding et al. [7] to account for aging in the SGR model framework.

There have been numerous studies on the effect of shear on aging dynamics [24-26]. Particularly relevant to our findings is the work by Ozon et al. [26] where it was reported that shear speeds up aging and that the acceleration factor is of the form $\exp \left(-\gamma / \gamma_{c}\right)$, where $\gamma_{c}$ is the critical strain. The authors argued that an Eyring-type model describes the effect of shear on aging. Our observations are different. Specifically, we find that deformation accelerates relaxation dynamics during application of shear, and that the acceleration factor is of the form $\exp \left(-\gamma^{2} / 2 x\right)$. Further, we show that this form is consistent with the strain dependence of elastic energy. Indeed, the remarkable agreement between the functional form of the experimentally determined $\tau(\gamma)$, [see fit in Fig. 2(b) and Eq. (2)] and the SGR model prediction [Figure 4(b), Eq. (6)] appears to validate the elastic energy-based framework used in the SGR model. The constant $k$ in Eq. (2) (obtained by fitting the SGR model to the data) is the effective spring constant for the system; it contains material-specific information about the interparticle interactions.

The appearance of a fast relaxation mode at high strain seems to be generic for our materials, but is not captured within the SGR framework. The possibility of a fast $\beta$ relaxation processes in soft glasses has been discussed by Sollich [6], however, as pointed out by the author such processes cannot be described within the low-frequency framework of the current SGR model. It can, in fact, be seen from Fig. 4(a) that the short time response in this model is always elastic with $G=1$ at all strains. Additionally, we note that whereas the experimental relaxation moduli are stretched exponential functions of time, the predicted moduli are power laws. We will discuss the origin of this discrepancy in a forthcoming article.

\section{CONCLUSION}

We have demonstrated both experimentally and theoretically that strain accelerates relaxation dynamics of selfsuspended suspensions of $\mathrm{SiO}_{2}$ nanoparticles densely grafted with polyisoprene chains, which manifest soft glassy rheology. To evaluate the generality of our findings, we performed similar experiments using suspensions of sterically stabilized $\mathrm{SiO}_{2}$-PEG nanoparticles dispersed in oligomeric polyethylene glycol (PEG) hosts, as well as using self-suspended suspensions created by densely grafting PEG to silica nanoparticles [27]. As shown in the supporting information, both systems manifest soft glassy rheology and exhibit strain-accelerated dynamics qualitatively similar to what is observed for selfsuspended $\mathrm{SiO}_{2}$-PI NIMs. Our findings therefore suggest that strain-induced acceleration of relaxation dynamics is a generic feature of many soft glasses. That the phenomena are also predicted within the SGR framework implies that it should be observed for all soft glasses governed by this framework.

\section{ACKNOWLEDGMENTS}

This work was supported by Award No. KUS-C1-018-02, made by King Abdullah University of Science and Technology (KAUST), and by the National Science Foundation, Award No. DMR-1006323. Facilities available though the Cornell Center for Materials Research (CCMR) were used for this study.
[1] H. Eyring, J. Chem. Phys. 4, 283 (1936).

[2] H. Lee, K. Paeng, S. F. Swallen, and M. D. Ediger, Science 323, 231 (2009).

[3] M. Warren and J. Rottler, Phys. Rev. Lett. 104, 205501 (2010).

[4] P. N. Pusey and W. van Megen, Nature (London) 320, 340 (1986).

[5] T. G. Mason and D. A. Weitz, Phys. Rev. Lett. 75, 2770 (1995).
[6] P. Sollich, Phys. Rev. E 58, 738 (1998); P. Sollich, F. Lequeux, P. Hebraud, and M. E. Cates, Phys. Rev. Lett. 78, 2020 (1997).

[7] S. M. Fielding, P. Sollich, and M. E. Cates, J. Rheol. 44, 323 (2000).

[8] V. Kobelev and K. S. Schwiezer, Phys. Rev. E 71, 021401 (2005). 
[9] Y. M. Joshi and G. R. Reddy, Phys. Rev. E 77, 021501 (2008).

[10] P. Agarwal, H. Qi, and L. A. Archer, Nano Lett. 10, 111 (2010).

[11] H. M. Wyss, K. Miyazaki, J. Mattsson, Z. Hu, D. R. Reichman, and D. A. Weitz, Phys. Rev. Lett. 98, 238303 (2007).

[12] H. Y. Yu and D. L. Koch, Langmuir, 26, 16801 (2010).

[13] K. N. Pham et al., J. Rheol. 52, 649 (2008); M. E. Hegelson, N. J. Wagner, and D. Vlassopoulos, ibid. 51, 297 (2007).

[14] J. L. Nugent, S. S. Moganty, and L. A. Archer, Adv. Mater. 22, 3677 (2010).

[15] Q. Zhang and L. A. Archer, Langmuir 18, 10435 (2002).

[16] G. Yin and M. J. Solomon, J. Rheol. 52, 785 (2008).

[17] C. Derec, G. Ducouret, A. Ajdari, and F. Lequeux, Phys. Rev. E 67, 061403 (2003).

[18] G. B. Mckenna, T. Narita, and F. Lequeux, J. Rheol. 53, 489 (2009).

[19] R. Bandopadhyay, P. H. Mohan, and Y. M. Joshi, Soft Matter 6, 1462 (2010).
[20] J. Mattson et al., Nature (London) 462, 83 (2009).

[21] M. Cloitre, R. Borrega, F. Monti, and L. Leibler, Phys. Rev. Lett. 90, 068303 (2003).

[22] P. A. O'Connell, G. B. Mckenna, Mech. Time-Depend. Mater 6, 207 (2002).

[23] M. Rubinstein, R. H. Colby, Polymer Physics (Oxford University Press, Oxford, 2003).

[24] V. Viasnoff and F. Lequeux, Phys. Rev. Lett. 89, 065701 (2002); V. Viasnoff, S. Jurine, and F. Lequeux, Farady Discuss 123, 253 (2003).

[25] D. Bonn, S. Tanase, B. Abou, H. Tanaka, and J. Meunier, Phys. Rev. Lett. 89, 015701 (2002).

[26] F. Ozon, T. Narita, A. Knaebel, G. Debregeas, P. Hebraud, and J. P. Munch, Phys. Rev. E 68, 032401 (2003).

[27] See supplemental material at [http://link.aps.org/supplemental/ 10.1103/PhysRevE.83.041402] for the experimental data on the oligomer- and self-suspended $\mathrm{SiO}_{2}$-PEG materials. 